\title{
ABORTO HABITUAL
}

\author{
DOCTOR Hernando EcheverRI V. \\ Trabajo presentado en el Seminario sobre Aborto que tuvo \\ lugar en la Sociedad Médica Quirúrgica ciel Atlántico en su \\ sesión de noviembre de 1956.
}

Me corresponde en turno hacer un compendio sobre A. H., mas como ya mis compañeros han hablado de las causas y tratamiento del aborto en general, necesariamente habré de incurrir en repeticiones enojosas que ruego se me perdonen.

El tema propiamente hablando corresponde al problema de infertilidad y pertenece, no ya al campo de la Medicina General sino más bien al de la Ginecología y Obstetricia, o mejor aún al de Esterilidad e Infertilidad, rama de la medicina que gana cada cía más adeptos.

Conviene, antes de entrar en materia, definir algunos térmiros: Infertilidad es la incapacidad para llevar a cabo el producto de la concepción, impotencia gestandi. Se entiende por aborto, según Javert (7), la terminación de todo embarazo antes de la 22 a semana que resulte en un feto de 500 grs. o menos. De 501 grs. en adelante se considera que el niño es viable.

En la definición del A. H. no hay un acuerdo completo, de ahí que las estadísticas no concuerden al mostrar los éxitos o fracasos de tal o cual tratamiento, porque mientras unos consideran que existe A. H. desde que haya habido dos abortos o más, otros, la mayoría, suben este número a tres. Parece que esto último es lo más aceptado, es decir, que para que haya $A$. $H$. se necesita el haber tenido tres o más abortos consecutivos, porque se presupone que en tres o más fracasos sucesivos, existe una causa común a todos ellos.

En el A. H. primario los 3 o más abortos consecutivos tienen lugar desde el primer embarazo. En el secundario vienen des- 
pués de uno o más fetos normales, Esta división es importante y hay que tenerla en cuenta al hacer cualquiera estadística, pues el pronóstico es más favorable en el secundario que en el primario.

Según el mismo Javert (7) se encuestra un caso de A. H. primario por cada 300 embarazos y uno secundario por cada 493. Randall (11) dice que de cada 100 embarazos hay siempre una pérdida inevitable de un $10 \%$ y un $1.4 \%$ de A. H. Esto demuestra que la incidencia de éste no es muy grande, de ahi que al médico general, y aun al especialista. les sea difícil adquirir experiencia en tales casos.

\section{ETIOPATOGENIA}

Me alargaría demasiado si entrara a analizar, o a enumerar siquiera, todas las causas que intervienen en el A. H., que en líneas generales no son otras que las del aborto común, tema que ya ha sido muy bien tratado por uno de mis colegas. Importa sí, hacer mención especial de algunas de ellas, sobre todo de las que han adquirido gran importancia en los últimos tiempos.

Recordemos, para claridad de la exposición, que las causas que pueden provocar aborto son ya de origen materno, paterno $\mathrm{u}$ ovular. $\mathrm{Y}$ las de origen materno se dividen, a su vez en generales y pélvicas.

\section{Causas generales de origen materno.}

\section{Deficiencias hormonales.}

Entre las causas generales de origen materno, la más llevada y traida en la actualidad es la hormonal. Como dice Calvin (1), mucho se ha ganado en este terreno desde que Frankel en 1903. comprobó que el cuerpo amarillo era necesario para la conservacićn del huevo en los conejos, y desde que Weinchert y Allen (1) en 1928 probaron que la progesterona prepara al endometrio para la recepción del huevo. En 1932 Reynolds y el mismo Allen, sigue diciendo Calvin (1) mostraron experimentalmente que la progesterona inhibe las contracciones uterinas producidas por la pituitrina. Después se agregó que esta hormona impide la destrucción de los estrógenos y facilita su conversión en estrona y estriol (15). Las cosas no quedaron allí, porque desde lo í trabajos de los esposos Smith (14), se encontró que la deficiencia estrogénica también podía ser causa de aborto. Finalmente la 
influencia de la hipófisis y el tiroides durante el embarazo ha sido ya demostrada.

Desde entonces acá. el uso y el abuso de los preparados hormonales continúa en el orden del día. Hoy se puede afirmar que, sobre todo en Colombia, no hay caso de amenaza de aborto o de A. $H$. que no se trate por hormonas. Esto se debe, en gran parte, a la falta de un tratamiento mejor, y en parte también a la escasez de laboratorios altamente especializados en la dosifización de hormonas. En los lugares donde si los hay, el alto costo de Estas reacciones impiden que se sistematicen. En otros países el tratamiento hormonal ha empezado a sentir el cortapisa de la crítica, pues hoy hay autores (1) que sostienen que el tratamiento hormonal en la amenaza de aborto, por ejemplo, sólo está indicado en un $3 \%$ de los casos.

\section{Deficiencia progesterónica en el aborto habitual.}

La deficiencia de esta hormona, como ya se anotó, fue la primera en cargar con la culpa en la producción de los abortos. Su empleo como curativo en el aborto común y como preventivo en el habitual, es sistemático entre nosotros no obstante su alto precio en el mercado. Esa terapéutica ha sido por lo general empírica, ya que en la mayoría de los casos se ignora si existe o no carencia de ella.

Cuando se perfeccionaron los métodos de dosificación del pregnandiol, que es su metabolito, se creyó dar con una guía más razonable para establecer un pronóstico y conducir un tratamiento. Sin embargo los resultados fueron un poco contradictorios en un principio, puesto que algunas veces no se observaron abortos con pregnandiol escaso, y en otras ocurrieron con niveles normales (15). Se llegó hasta citar casos con valores persistentemente negativos que habían llevado a término el embarazo (6). A aumentar esta confusión contribuyó la afirmación de Hamblen (4), quien dice que los valores de pregnandiol no suben con la medicación progesterónica.

No obstante todo esto, no se debe olvidar que Hughes sostiene, según shoeneck (13), que las enfermas con pregnandiol bajo que llegan a término, es porque tienen niveles altos de gonadotrofinas. Por otra parte Janow (6) mostró con estadísticas que la prueba del pregnandiol sí tiene valor para el pronóstico, puesto que en la amenaza de aborto con resultados positivos, el 
embarazo se lleva a término en un $89 \%$ de los casos, mientras que los resultados persistentemente negativos dan un, 95\% de abortos. En el aborto habitual, Vaux y Rakoff (15) encontraron deficiencia de pregnandiol en un $53 \%$ de los casos.

Fuera del pregnandiol se han ideado otras pruebas para diagnosticar la deficiencia progesterónica durante el embarazo. Una de ellas es el frote vaginal, en el cual deben desaparecer las células cornificadas cuando hay una buena producción progesterónica. Randall (11) en 1955 estudiando este método llegó a las siguientes conclusiones: En la amenaza de aborto un frote normal progesterónico indica resultados favorables en un $92.6 \%$ de los casos. El mismo frote en el A. H. da un $85 \%$. Cuando hay deficiencia (aumento de cornificación), en el primer caso se obtiene un $48.7 \%$. Pero si se trata de A. $\mathrm{H}$. con un mal frote los resultados bajan a 0 , especialmente si la cornificación excede a un $35 \%$.

Para investigar la deficiencia progesterónica está de moda ahora un método más sencillo que el anterior: Se trata de la cristalización en forma de helecho del moco cervical, fenómeno que se debe, dicho sea de paso, a la presencia del cloruro de sodio depositada allí por los estrógenos y que fue descrito por primera vez por Papanicolau (10) en 1946.

Si se extrae moco cervical con una pipeta, se extiende sobre una lámina, se deja secar a la temperatura ambiente o mejor con una lámpara de alcohol, y se mira al microscopio, se nota la formación de pequeños cristales que se ordenan espontáneamente como ramas de un helecho, sobre todo cuando la enferma está cerca o durante el período de ovulación. Campos da Paz (2) demostró que cuando se forman esta clase de figuras, el moco cervical se deja penetrar fácilmente por los espermatozoides y es a la vez un buen signo de funcionamiento estrogénico. A medida oue va pasando el tiempo de la ovulación las cristalizaciones van ciesapareciendo paulatinamente y ya durante el 26 o día no deben encontrarse. Su presencia para esta época significa, o que no ha habido ovulación, o que la respuesta progesterónica no ha sido satisfactoria (18).

Zondeck y col. (19) (20) extendieron este método al diagnóstico precoz del embarazo y al pronóstico con respecto al aborto. En presencia de un retardo menstrual la ausencia de helechos está en favor de un embarazo; si las arborizaciones existen, se trata de un simple retardo o de un embarazo con 
ఓmenaza de aborto por deficiencia progesterónica. Conviene agregar que el método, aunque parece sencillo, no está exento de cificultades, pues existen arborizaciones atípicas o falso' positivos, cuya interpretación requiere alguna práctica.

\section{Deficiencia de estrógenos como causa de A. H.}

Ya dijimos que los esposos Smith (14) fueron los primeros en señalar la deficiencia estrogénica como causa de A. H. Mucho se ha escrito desde entonces en favor y en contra de esta teoría. Hoy se admite que los estrógenos preparan el endometrio para la acción de la progesterona, la llamada fase proliferativa. Está igualmente comprobado que el cuerpo lúteo produce estrógenos a la par que la progesterona y que ambas hormonas siguen una curva ascendente y más o menos paralela durante el embarazo (15). Hasta hace poco se creyó que los estrógenos poseían una acción antagónica a la de la progesterona con respecto a la fibra muscular uterina, excitándola en vez de aquietarla, de ahí que estaban contraindicados durante el embarazo. Los Smith (14) demostraron que durante este período no existe tal antagonismo, pues el útero tolera cantidades enormes de esta hormona. Finalmente la escuela de los Smith (14) sostienen que los estrógenos ayudan a la conservación del cuerpo lúteo, favorecen la utilización de la progesterona y aumentan su producción. Esto último no ha podido demostrarse experimentalmente.

Pero los estrógenos no sólo cooperan en la conservación del cuerpo lúteo irrigándolo mejor, sino que provocan el crecimiento ce las arterias espirales de la caduca, para que habiendo una buena irrigación de esta membrana, haya un buen corión, lo que a su vez redunda en una mayor producción de gonadotrofinas coriónicas y por consiguiente de progesterona y de estrógenos. Los estrógenos son pues hormonas cuya acción se ejerce principalmente sobre los vasos de la esfera genital, especialmente sobre las arterias espirales, ya sean del ovario, endometrio, caduca o placenta, aumentando su calibre y su longitud con el fin ce provocar un mayor aporte de sustancias nutritivas al embrión. Sobre esto volveremos más adelante al tratar de las perturbaciones del endomertio como causa de aborto.

Volviendo al A. H. es interesante anotar que Vaux y Rakoff (15) encontraron que los estrógenos urinarios están disminuídos en esta entidad en un $67 \%$ de los casos. Dicen, además, que los estrógenos de la sangre son más constantes y fáciles de dosificar 
y constituyen un buen índice para el pronóstico, sobre todo si coinciden con las dosificaciones del pregnandiol.

\section{Gonadotrofinas.}

Como todos sabemos las gonadotrofinas son necesarias para el ciclo ovárico normal, pues excitan la producción de estrógenos y progesterona a su debido tiempo. Durante el embarazo desempeñan el mismo papel; pero como se necesita un aumento de estrógenos y de progesterona, se requiere también una buena cantidad de gonadotrofinas, para esto el corión fetal viene a ayudar a la hipófisis en los primeros días.

Los casos de A. H. debidos a deficiencia o exceso de gonadotrofinas son más bien raros. Vaux y Rakoff (15) las enəuentran normales en un $86 \%$ de los casos, disminuídas en un $7 \%$ y aumentadas en otro tanto. El exceso de gonadotrofinas habla en favor de una insuficiecia del ovario, que es incapaz de responder a su estímulo. La escasez, en cambio, debe interpretarse como un defecto de la hipófisis. En ambos casos el pronóstico es malo.

\section{Avitaminosis.}

Entre las causas generales de origen materno se ha destacado últimamente la avitaminosis. No hablaremos de la importancia de la vitamina A para el buen funcionamiento endometrial. pues es la misma que tiene para todos los epitelios. Parece que la vitamina $\mathrm{B}$ y su complejo son necesarios para el metabolismo de los estrógenos en el hígado. La vitamina $\mathrm{E}$. cuyo papel en la esterilidad e infertilidad de los animales ha sido demostrada, no ha dado los resultados que se esperaban en la especie humana.

Merecen mención especial la vitamina C y los compuestos citroflavonoides, o complejo de vitamina $\mathrm{P}$, cuya carencia influye en la génesis de la hemorragia según la escuela de Javert $y$ col. (7), quienes encontraron deficiencia de ellas en el A. H. La carencia de vitamina $K$ fue acusada en un principio por la misma escuela, pero con mejores métodos de laboratorio vieron que el tiempo de la protombina no se alteraba en estas enfermas.

Hughes y col. (5) también han llamado la atención sobre la riqueza del endometrio en vitamina C. Al final del ciclo, dicen, se encuentra en cantidades que fluctuan entre 164 y 168 mlgrs. 
por gramo de mucosa. Agregan que la acción que pueda tener esta vitamina en la implantación del huevo todava no está muy clara.

\section{Psiconeurosis.}

No podemos terminar el análisis de las causas generales de origen materno, sin antes anotar la gran influencia que tienen las psiconeurosis en el A. H. Por todos es conocida la importancia del factor psicogenético en medicina interna y hoy tenemos enfermedades ginecológicas de origen psiconeurótico así como casos de esterilidad e infertilidad del mismo origen.

Con el fin de estudiar mejor este factor, el New York Hospital abrió últimamente una clínica psiquiátrica para tratar esta clase de enfermas. Justamente hace poco acaba de presentar sus primeros resultados.

Edward Mann (9), autor de esta publicación, hace resaltar el hecho de que con cualquier tratamiento, en manos de un especialista avezado, se obtienen resultados bastante satisfactocios. Esto hace pensar, agrega, que el éxito así obtenido depende más de la personalidad del médico tratante, que conciente o inconcientemente ha logrado influenciar la enferma, que de la clase de droga usada.

Las abortadoras habituales, según él, suelen tener algunas peculiaridades psiquicas que les son comunes. Se trata por so general de una sola hija, o de la única mujer en la familia, o bien de la hija mayor o de la menor. La madre ejerce casi siempre una especie de matriarcado que hace que la hija se levante dependiendo siempre de ella y conserve una personalidad emotiva muy cerca de la infantil. El padre, ya sea por sus ocupaciones o por sus vicios, ha intervenido poco en la formación del carácter de sus hijos.

En términos generales se puede afirmar, pues, que no hay un caso de A. $\mathrm{H}$. en el cual no intervengan el factor psiconeurótico, sea éste adquirido desde la niñez o provocado por frustraciones sucesivas en el curso de la vida. De ahí que hoy en día la causa psicogenética ha adquirido gran importancia en la entidad que estamos comentando. 


\section{CAUSAS PELVICAS}

\section{Defectos del endometrio.}

Gracias a los trabajos de Hughes y col. (5) conocemos hoy mejor el papel que desempeñan los defectos endometriales en el A. H. Como se sabe, el endometrio está destinado a procurar un sitio apropiado para la nidación del huevo, proceso fisiológico que eśtá regido por el sistema nervioso y el sistema endocrino.

Según Hughes y col. (5), antes que el blastocito asuma su función hemotrófica, primeras 2 a 3 semanas, su alimento debe ser suministrado por las secreciones glandulares del endometrio, por el flúido extracelular y por los detritus que se forman durante la implantación: la llamada "leche uterina". Durante este petíodo, pues, el endometrio necesita producir una clase de alimento que sea a propósito para el crecimiento rápido del blastocito, de lo contrario pueden sobrevenir daños irreparables para el huevo.

La substancia que más se presta para este fin es la glucosa. Esta es llevada al endometrio por los vasos sanguíneos, su abundancia o escasez dependen del buen o mal desarrollo del sistema vascular. La glucosa se almacena en el endometrio al estado de glicógeno y pasa, por tanto, por las diferentes fases de toda glicogénesis, es decir, que primero debe fosforizarse y formar el fosfato de glucosa, para luégo polimerizarse y convertirse el glicógeno. Cuando el huevo se implanta, el glicógeno, que no puede ser utilizado como tal debe descomponerse de nuevo para ser transformado en glucosa. Estas reacciones histoquímicas necesitan de dos clases de encimas: la fosfatasa alcalina, que es la que ayuda a la transformación de glucosa en glicógeno, y las encinas líticas, que son las que parten la molécula de glicógeno para convertirlo de nuevo en glucosa.

La fosfatasa alcalina, seguimos citando a Hughes (5), aparece en pequeñas cantidades en las arteriolas del endometrio poco antes de la ovulación. Ya durante ésta se encuentra acumulada en el protoplasma y núcleos de las células glandulares en gran cantidad. A medida que el glicógeno se va formando, la fosfatasa va disminuyendo gradualmente de las células y al final del ciclo, $26^{\circ}$ días, ya se encuentra en mayor cantidad en el lumen glandular. A la par con este proceso va el de la formación de glicógeno, que en un principio se va acumluando bajo la forma de glóbulos en ia base de las células, rechazando sus núcleos hacia la periferia, 
para ocupar más tarde, durante el período secretorio, la parte distal de éstas, rechazando de nuevo sus núcleos hacia la base. En este momento es cuando intervienen las encimas líticas, que también habian ido aumentando a medida que el ciclo se aproximaba a su fin. $\mathrm{Y}$ así queda listo el endometrio para la recepción del huevo.

Hughes y col. (5), mediante coloraciones especiales, estudiaron la glicogénesis del endometrio en las abortadoras habituales y hallaron que en un 58\% de ellas este proceso era deficiente. Los hallazgos más notorios que hicieron en las biopsias tomadas durante el 260 día del ciclo de estas enfermas fueron: escasez de glicógeno, escasez de encimas líticas, y endometrio en fase proliferativa. En los abortos de estas mismas enfermas encontraron trombosis con hemorragia y necrosis de la decidua, atrofia de las vellosidades coriales, ausencia o falta de desarrollo de las células de Langhans, separación prematura de la placenta y placenta circunvalata.

Deformidades de la cavidad uterina.

Traumatismo del coito.

No queremos terminar el análisis de las causas de origen pélvico, sin antes hacer resaltar la importancia que han adquirido las deformidades de la cavidad uterina y los traumatismos del coito en la génesis del A. H. Por no alargarnos demasiado prescindiremos de las demás causas de este origen.

Con el uso más frecuente de los Rayos $\mathrm{X}$ en ginecología, se ha venido observando que las deformidades de la cavidad uterina aumentan su frecuencia en el A. H., hasta el punto que hay autores (3) que recomiendan la histerografía sistemática en estos casos. Las deformidades pueden ser, ya congénitas como útero duplex, bicorne, etc., ya adquiridas como en los pólipos y miomas itracavitarios.

Cuanto al traumatismo del coito, Javert y col. (7), mediante un interrogatorio muy detenido de las enfermas, observaron que casi siempre precede a la hemorragia, de ahí que ellos prohiban las relaciones sexuales durante todo el embarazo. Insistimos sobre esto porque entre nosotros se olvida, tanto por parte del médico como de los cónyuges, dar suficiente importancia a este factor. 


\section{CAUSA OVULAR}

La causa ovular ha sido puesta al día por Wall y Herting (16), se trata del factor llamado "defecto del plasma germinal". Su importancia se dedujo del estudio de los huevos fecundados de pocos días, hallados en las trompas o en la cavidad uterina, durante las histerectomías. Herting encontró que en un $46 \%$ de Ios abortos, se trata de huevos anormales, entendiéndose por huevo anormal todo aquel en que puede apreciarse algún defecto ya sea macro o microscópicamente hablando. Mall, citado por Rutheford (12), halló un 75\% de huevos anormales en las enfermas que tenían menos de un mes de embarazo; de 2 meses un $50 \%$, y de 3,4 a 5 sólo un $15 \%$.

En estos casos el papel que juega la anormalidad del huevo en sí, "plasma germinal defectuoso", o las deficiencias del medio ambiente, endometrio, no está bien determinado, entre otras cosas porque el estudio del huevo no fecundado es difícil por falta de material. El papel del espermatozoide tampoco está bien claro, aunque hoy se admite que una anormalidad mayor de un $20 \%$ en el espermograma predispone a la producción de huevos anormales. Por otra parte el porcentaje de huevos defectuosos implantados en la trompa es muchísimo mayor que los hallados en la cavidad uterina, lo cual está en favor de la enorme influencia que tiene el medio ambiente (12).

\section{TRATAMIENTO}

De 10 que llevamos expuesto se deduce que para dirigir bien un tratamiento en el A. H. es necesario conocer, en cuanto sea posible, la causa que está provocando los abortos, y para esto es necesario un examen concienzudo no sólo de la paciente sino de su cónyuge. Una vez en poder de antecedentes y datos generales de la pareja, se entra al estudio de las hormonas, endometrio, metabolismo basal, etc., en la mujer, y el de un espermograma en el hombre.

El tratamiento en sí es sobre todo preventivo. En primer lugar todo médico general, o especialista, debe ser muy cuidadoso con las enfermas que han tenido 1 o 2 abortos, tratando por todos los medios que estén a su alcance de evitar que su enferma se convierta en una abortadora habitual. La importancia de esto se desprende de las conclusiones de Malpas (8) en su estudio sobre más de 6.000 abortos, según las cuales toda paciente que ha tenido un aborto tiene 78 probabilidades de 
llevar a término un segundo embarazo; si ha tenido 2 este porcentaje baja a 62; cuando ha habido 3 abortos consecutivos las probabilidades de éxito sólo son de $27 \%$, y en las de 4 o más abortos se reducen a $10 \%$.

Tratamiento hormonal.-Una vez bien estudiada la enferma, o mejor la pareja, si se decide por un tratamiento hormonal existen varios caminos a seguir, según la experiencia de cada cual. Ante todo, si los resultados de laboratorio favorecen a tal o cual hormona, es obvio que ese tratamiento será el preferencial. Pero hablando en términos generales se puede afirmar que cualquiera de las hormonas, bien manejada, ha dado buenos resultados en manos de los expertos. Ya dijimos que en esto tiene mucha parte la psicología de la enferma y la personalidad del médico tratante. En todo caso los éxitos que deben esperarse fluctúan entre un 70 a un $80 \%$. Un $90 \%$ equivaldría a un $100 \%$. pues normalmente hay siempre un $10 \%$ de embarazos que se pierden.

Todo tratamiento, hormona $₫$ no, debe empezarse antes que aparezcan los síntomas de aborto, pues cuando ya hay sangre es muy frecuente que el embrión esté muerto. Hughes y col. (5) van todavía más allá y lo empiezan antes del embarazo, en otros términos, preparan al endometrio para la recepción del huevo. El fin que se proponen es provocar un buen aporte de glucosa, fosfatasa alcalina y encimas líticas, la llamada "eche uterina" de que ya hablamos. Para esto usan pequeñas dosis de estrógenos ciurante los primeros 20 días del ciclo menstrual, teniendo buen cuidado de no excederse en las dosis porque de lo contrario se inhibe la ovulación. Los estrógenos a pequeñas dosis producen una buena fase proliferativa con el consiguiente aumento del volumen y longitud de las arterias espirales y de la red capilar que rodea las glándulas provocando así un aumento de glicógeno y fosfatasa alcalina como lo demuestran las biopsias subsiguientes. Aconsejan continuar el tratamiento por tres meses antes de autorizar un embarazo.

Para terminar con este breve resumen sobre el tratamiento hormonal, diremos que unos prefieren la progesterona, otros los estrógenos, y que la tendencia actual es la de combinar ambas hormonas. Robert B. Wilson (17) acaba de publicar un trabajo en el cual aboga por el uso de tres hormonas: progesterona, estrógenos y gonadotrofina coriónica. Los resultados obtenidos̃ 
con este tratamiento han sido de un $100 \%$ de éxitos, aunque como él mismo anota, el número de casos es todavía muy pequeño.

Finalmente diremos que la medicación tiroidea resulta un magnífico coadyuvante, sobre todo si el M. B. es bajo.

\section{Tratamiento por vitaminas.}

Ya vimos que Javert y col. (7) hallaron que en el A. H. había con frecuencia deficiencia de vitaminas $\mathrm{C}$ y complejo P. El tratamiento aconsejado por ellos consiste en la administración de estas dos vitaminas, junto con una serie de medidas tales como alimentación bien compensada, abstención del coito, higiene mental o psicoterapia si es necesario. No exigen reposo en cama sino durante los períodos de amenaza de aborto, y eso por corto tiempo. NO USAN NINGUNA CLASE DE HORMONAS. LOS éxitos obtenidos con este tratamiento fueron de un $80 \%$, en contraposición con un $26.4 \%$ de las enfermas testigo.

Me he abstenido adrede de dar dosificaciones tanto de hormonas como de vitaminas, ya que este tema ha sido tratado, con lujo de detalles, por mi colega. Quiero solamente añadir, para terminar, que no existe un tratamiento específico del A. H. La escogencia de éste depende del examen de la enferma y de la experiencia de cada cual. Todo tratamiento, sea hormonal, vitamínico, o combinado, debe complementarse con medidas higiénicas generales y con psicoterapia, como lo aconseja la mayoría de los autores.

\section{BIBLIOGRAFIA}

1. CALviN, E. D. y col. Am. J. Obst. y Gynec. 59: 1208, 1950.

2. DA PAZ, CAMPOS.-Fertil .y Steril. 4: 137, 1953.

3. HALBRHECH, I.-Fertil. y Steril. 4: 272, 1953.

4. HAMBLEN.-Citado por Vaux y Rakoff (15).

5. HUGHES, E. C.-Am. J. Obst. y Gynec. 59: 1292, 1950.

6. JANOW M.-Am. J. Obst. y Gynec. 59 1160, 1950.

7. JAVERT CARL T.-Am. J. Obst y Gynec. 57: 878, 1949.

8. MALPAS.-Citado por Rutherford (12).

9. MANN, EDWARD C.-Obst. y Gyn. 7: 589, 1956.

10. PAPANICOLAU GEORGE N.--Am. J. Obst. y Gynec. 51: 316, 1946.

11. RANDALL, CLYDE L. y col.-Am. J. Obst. y Gynec. 69: 643, 1955.

12. RUTHERFORD, R. N.-Am. J. Obst. y Gynec. 51: 652, 1946.

13. SHOENECK, F. J.-Am. J. Obst. y Gynec. 69: 657, 1955.

14. SMITH Y SMITH.-Am. J. Obst. y Gynec. 51: 411, 1946.

15. VAUX, N. W. RAKOFF, A. E.-Am4 J. Obst. y Gynec. 50: 353, 1945.

16. WALL, R. L. HERTING, A. T.-Am. J. Obst. y Gynec. 56: 1127, 1948.

17. WILSON, R. B.-Am. J. Obst y Gynec. 69: 614, 1955.

18. ZONDEK, B. y col.-Obst. y Gyn. 3: 463, 1954

19. ZONDEK, B. y col.-Fertil y Steril. 6: 523, 1955.

20. ZONDEK, B.-Int. J. of Fertility 1: 225, 1956. 